\title{
Innovative dressing and securement of tunneled central venous access devices in pediatrics: a pilot randomized controlled trial
}

Amanda J. Ullman ${ }^{1,2^{*}}$, Tricia Kleidon ${ }^{2,3}$, Victoria Gibson ${ }^{2,3}$, Craig A. McBride ${ }^{2,4,5}$, Gabor Mihala ${ }^{2,5,6}$, Marie Cooke and Claire M. Rickard ${ }^{1,2}$

\begin{abstract}
Background: Central venous access device (CVAD) associated complications are a preventable source of patient harm, frequently resulting in morbidity and delays to vital treatment. Dressing and securement products are used to prevent infectious and mechanical complications, however current complication rates suggest customary practices are inadequate. The aim of this study was to evaluate the feasibility of launching a full-scale randomized controlled efficacy trial of innovative dressing and securement products for pediatric tunneled CVAD to prevent complication and failure.

Methods: An external, pilot, four-group randomized controlled trial of standard care (bordered polyurethane dressing and suture), in comparison to integrated securement-dressing, suture-less securement device, and tissue adhesive was undertaken across two large, tertiary referral pediatric hospitals in Australia. Forty-eight pediatric participants with newly inserted tunneled CVADs were consecutively recruited. The primary outcome of study feasibility was established by elements of eligibility, recruitment, attrition, protocol adherence, missing data, parent and healthcare staff satisfaction and acceptability, and effect size estimates for CVAD failure (cessation of function prior to completion of treatment) and complication (associated bloodstream infection, thrombosis, breakage, dislodgement or occlusion). Dressing integrity, product costs and site complications were also examined.
\end{abstract}

Results: Protocol feasibility was established. CVAD failure was: 17\% (2/12) integrated securement-dressing; 8\% (1/13) suture-less securement device; 0\% tissue adhesive (0/12); and, 0\% standard care (0/11). CVAD complications were: 15\% (2/13) suture-less securement device (CVAD associated bloodstream infection, and occlusion and partial dislodgement); $8 \%(1 / 12)$ integrated securement-dressing (partial dislodgement); $0 \%$ tissue adhesive (0/12); and, $0 \%$ standard care (0/ 11). One CVAD-associated bloodstream infection occurred, within the suture-less securement device group. Overall satisfaction was highest in the integrated securement-dressing (mean 8.5/10; standard deviation 1.2). Improved dressing integrity was evident in the intervention arms, with the integrated securement-dressing associated with prolonged time to first dressing change (mean days 3.5).

Conclusions: Improving the security and dressing integrity of tunneled CVADs is likely to improve outcomes for pediatric patients. Further research is necessary to identify novel, effective CVAD securement to reduce complications, and provide reliable vascular access for children.

(Continued on next page)

\footnotetext{
* Correspondence: a.ullman@griffith.edu.au

${ }^{1}$ School of Nursing and Midwifery, Griffith University, Nathan, Queensland,

Australia

${ }^{2}$ Alliance for Vascular Access Teaching and Research Group, Menzies Health

Institute Queensland, Nathan, Queensland, Australia

Full list of author information is available at the end of the article
} 
(Continued from previous page)

Trial registration: ACTRN12614000280606; prospectively registered on 17/03/2014.

Keywords: Central venous catheter, Dressing, Randomized controlled trial, Evidence-based care, Pediatrics

\section{Background}

Children undergoing treatment for oncological and hematological conditions are some of the most vulnerable patients in hospital settings. Functioning, complicationfree vascular access for anti-cancer therapies, antibiotics, nutrition and blood sampling is vital to their treatment and survival. Tunneled, cuffed central venous access devices (CVADs), commonly referred to by their trade names, Hickman ${ }^{\bullet}$ or Broviac ${ }^{\bullet}$ catheters, are inserted for children requiring multiple or frequent infusions of irritant or vesicant fluids over prolonged periods of time $(\geq 3$ months) [1]. The pediatric population requiring this type of central device is primarily receiving treatment for oncological (e.g. neuroblastoma), hematological (e.g. aplastic anemia) or gastroenterological (e.g. intestinal failure) conditions [2,3].

Tunneled, cuffed CVADs incorporate a short Dacron cuff designed to inhibit ascending migration of organisms from the skin, and to stimulate tissue growth around the cuff to anchor the device [4]. However Dacron cuff adhesion takes up to four weeks $[4,5]$, longer when the patient is compromised, placing the CVAD at significant risk of dislodgement or infection prior to that time. While historically considered a 'safe' device, a recent meta-analysis [6] demonstrated $29 \%$ of pediatric tunneled cuffed CVADs failed prior to completion of therapy (0.86 per 1,000 catheter days), with $20 \%$ developing a CVAD-associated bloodstream infection (BSI) (1.13 per 1,000 catheter days), and 7\% dislodging completely ( 0.24 per 1,000 catheter days).

A key strategy to decrease the risk of CVADassociated complication is to ensure the insertion wound is adequately covered to prevent infection, and the device secured to prevent internal and external motion [7]. Traditionally CVAD insertion sites were covered with sterile gauze and tape, with polyurethane dressings becoming prominent practice in the 1990s [8]. The devices are also internally and/or externally sutured, with these sutures removed, or dissolving, at around 7-10 days. The current failure and complication rates associated with CVADs suggest that habitual practices of their dressing and securement are inadequate.

A recent Cochrane systematic review [9] highlighted the dearth of literature to support clinical decision making in the area of CVAD securement, considering the range of available products. Previous randomized controlled trials (RCTs) of securement and dressing products for tunneled, cuffed CVAD are dated $[10,11]$ and have limited their study population to adults $[10,12-$ 14]. No previous RCTs have focused on children or examined the issue of CVAD security. The physiology and pathophysiology of children requiring these devices necessitates a specialist focus [15].

Newer CVAD securement and dressing products are available, which may be superior to traditional methods. Integrated securement-dressings (ISDs) incorporate a strengthened securement system across the entire polyurethane dressing, over and underneath the CVAD body [7]. ISDs also surround the polyurethane with an absorbent layer, to maintain dressing integrity when exposed to insertion site exudate. Suture-less securement devices (SSD) comprise of soft footplates with fastening clasps of hard plastic or soft Velcro to reduce movement and catheter rotation [16]. Tissue adhesive (TA), a medical grade 'superglue' (cyanoacrylate), can provide strength and hemostatic properties [17], which may be beneficial for patients experiencing large amounts of post-insertion exudate due to underlying pathologies. However, it is not known whether these new products are more effective at reducing pediatric CVAD failure and complication, in comparison to traditional care. In order to reduce CVAD associated complications in the pediatric population, RCTs of CVAD securement products are necessary to provide true estimates of relative effectiveness and inform practice [18]. Prior to undertaking large efficacy trials, external pilot studies are necessary to examine issues of research feasibility including intervention acceptability, compliance and recruitment [18].

\section{Methods \\ Aims}

The primary aim of this research was to evaluate the feasibility of launching a full-scale randomized controlled efficacy trial of pediatric tunneled CVAD securement and dressing, using pre-defined feasibility criteria for recruitment, retention, protocol fidelity and product acceptability. The secondary aim was to compare the effectiveness of dressings and securement products on tunneled CVAD complications and failure due to infection, occlusion, dislodgement, thrombosis, or breakage, for children in acute care facilities.

\section{Design}

This study was a four-arm, external pilot randomized controlled trial. Prior to study commencement the trial was registered with the Australian Clinical Trial Registry 
(ACTRN12614000280606), including a published protocol [19].

\section{Study setting}

The trial was commenced at the Royal Children's Hospital, Brisbane, Australia; and, after local hospital mergers, completed at the larger Lady Cilento Children's Hospital, Brisbane, Australia. These are tertiary level, specialist pediatric teaching hospitals, providing health services to children and young people from birth to 18 years of age throughout Queensland, northern New South Wales and the Pacific Rim.

\section{Sample}

Participants who met the inclusion criteria were consecutively recruited: requiring a tunneled, cuffed CVAD; less than 18 years of age; would remain hospital inpatients for greater than $24 \mathrm{~h}$; and informed consent to participate. Patients were not eligible if they had a current bloodstream infection, consent givers were nonEnglish speakers without an interpreter, the CVADs were to be inserted through diseased, burned, scarred or extremely diaphoretic skin, had a known allergy to the study products, had a current skin tear, or had previously been enrolled in the study within the current hospital admission.

Twelve participants were recruited per intervention group, with a final sample of 48 participants. These sample size calculations were developed in accordance with the recommendations by SA Julious [20] and M Hertzog [21]; to facilitate accurate estimates of effect size while minimizing unnecessary costs, time and recruitment of future definitive study participants, where little data are available to base a sample size calculation.

\section{Interventions}

Participants were randomized to receive CVAD dressing and securement by:

- Standard care: Suture (Prolene ${ }^{\circ}$; Ethicon, New Jersey); and bordered polyurethane (BPU) dressing (Tegaderm ${ }^{\bullet} 1655$ or 1616 dependent upon participant size; 3M, St Paul);

- Suture-less securement device (SSD): Suture (Prolene ${ }^{\circ}$ Ethicon, New Jersey) (suture was deemed necessary due to large tunnel wound); Suture-less securement device (staff preference of StatLock ${ }^{\circ}$ VFDSSP; Bard, Georgia or GripLok ${ }^{\circ} 3601$ CVC; TIDI, Neenah WI); and BPU dressing (Tegaderm ${ }^{\circ}$ 1655 or 1616; 3M, St Paul);

- Tissue adhesive (TA): One-two drops of Tissue adhesive (Histoacryl ${ }^{\circ}$; B. Braun, Germany) at exit wound and under catheter bifurcation; and BPU dressing (Tegaderm ${ }^{\circ} 1655$ or 1616; 3M, St Paul);
- Integrated securement-dressings (ISD): Suture (Prolene ${ }^{\circ}$ : Ethicon, New Jersey); and ISD (SorbaView SHIELD ${ }^{\circ}$ SV254; Centurion Medical Products, Williamston).

The application of these intervention arms can be seen in Fig. 1. The intervention arms were developed taking into consideration current local practice, best available evidence and the safety of all participants.

\section{Outcomes}

The primary outcome was feasibility of a full efficacy trial, established by composite analysis of elements of eligibility, recruitment, attrition, protocol adherence, missing data, parent and healthcare staff satisfaction, and effect size estimates to allow sample size calculations [21-23]. Sample size estimates were to be based upon the proportion of CVAD failure (cessation of catheter function prior to completion of therapy [6]), and CVAD complication (a composite of CVAD-associated BSI (CABSI), local infection, occlusion, dislodgement, venous thrombosis and breakage). Each CVAD complication was defined in accordance with best practice guidance $[3,6,24,25]$.

Secondary outcomes included the individual CVAD complications, CVAD-related BSI [24], securementdressing failure, time to first dressing change, skin complications and direct product costs [26]. Full definitions of the primary and secondary outcomes can be found within the published protocol [19]. Diagnoses of CABSI and CVAD-related BSI outcomes were by an independent, blinded infectious diseases specialist. Similarly, ultrasound for the identification of symptomatic venous thrombosis was requested by the clinical team coordinating the participants' care, with diagnosis made by an independent, blinded radiologist using standard departmental protocols.

\section{Study Procedures}

The research nurse $(\mathrm{ReN})$ screened patients daily via theatre bookings, then obtained written informed consent, and performed randomization. Randomization was evenly distributed 1:1:1:1 between study groups with randomly varying block sizes, using a web-based service (https://www151.griffith.edu.au/random) that ensured concealment. The ReN checked patients daily to inspect the CVAD and dressing securement products, collect data, and to ensure safety of the study participants. Participants were included in the trial until four weeks after CVAD insertion, or to study withdrawal, removal of the CVAD, or hospital discharge, if these occurred before four weeks. CVAD securement and dressings were not amenable to blinding of patients, clinical staff or ReNs. 

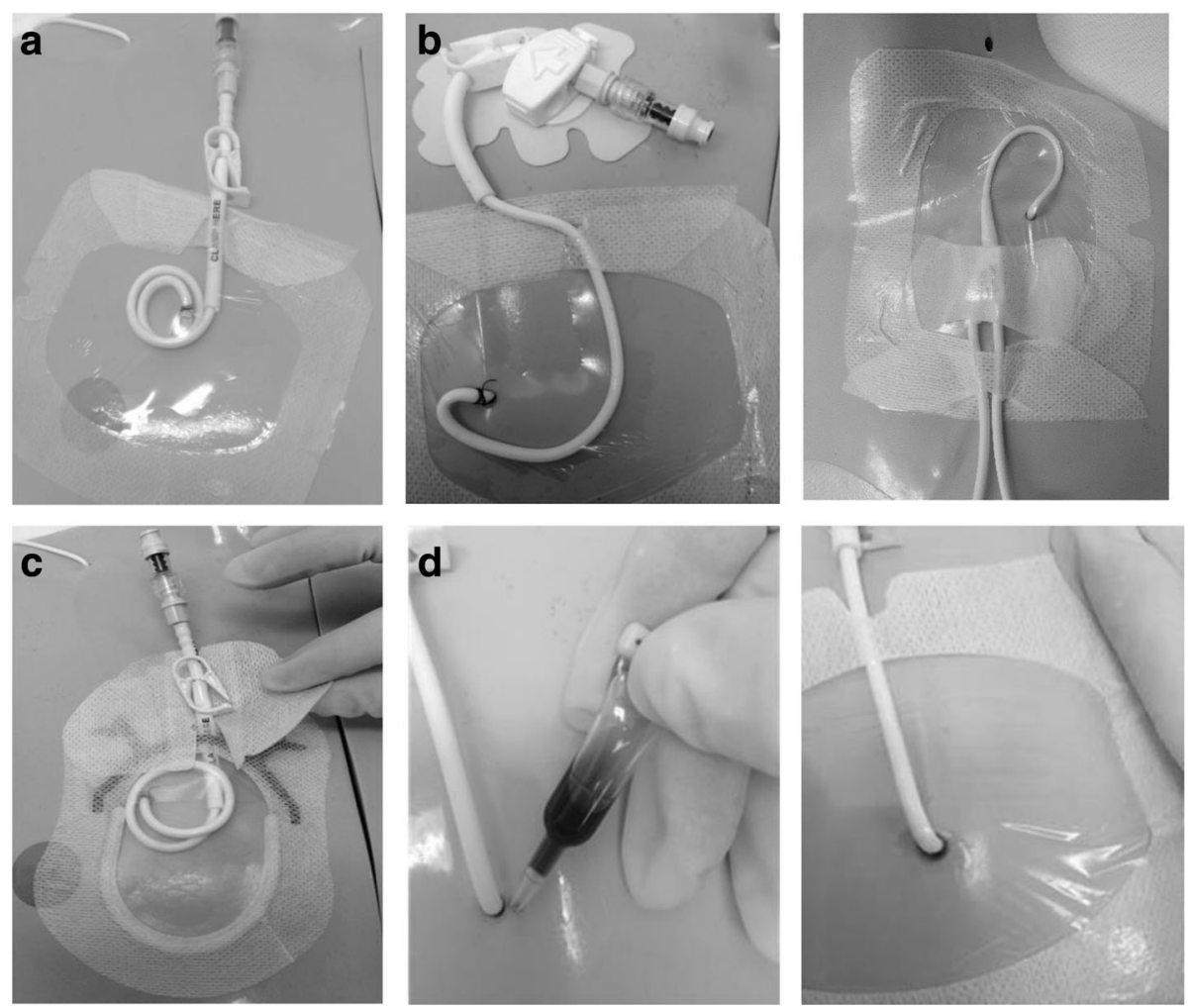

Fig. 1 Intervention products. a Bordered polyurethane and suture; b Bordered polyurethane, suture and suture-less securement device; $\mathbf{c}$ Integrated securement dressing and suture; $\mathbf{d}$ Bordered polyurethane and tissue adhesive (no suture)

De-identified data collection was undertaken via REDCap (Research Electronic Data CAPture http://project-redcap.org/). The ReN collected data on primary and secondary outcomes using the pre-defined criteria. Demographic data were collected to assess success of randomization, describe the participant group and enable comparisons to inform future generalizability. Data were also collected regarding clinical characteristics known to increase the risk of CVAD complication and dressing integrity, including age, comorbidities, immune-compromise, CVAD utility, skin condition, insertion site and technique [2, 3, 27-31]. At CVAD insertion and removal (or within $24 \mathrm{~h}$ ), the ReN asked the parents or caregivers, and healthcare staff for their perceived satisfaction with the intervention products (numeric rating scale $0-10$ with increased positivity with higher scores).

\section{CVAD procedures}

All non-antimicrobial tunneled, cuffed CVADs $\left(\right.$ Cook $^{\odot}$; Cook Medical; Bloomington) were inserted in an operating theatre by a qualified consultant pediatric surgeon, or a surgeon in an approved pediatric surgical program, and managed by clinical staff in accordance with state and hospital policy [32-34]. The inserter chose the CVAD characteristics based on clinical judgement of patient needs, and then applied the allocated products [35]. Local hospital policy directed site decontamination at insertion with aqueous Betadine (10\% povidoneiodine) and no routine antibiotic prophylaxis at insertion [34]. To maximize generalizability, clinical nursing staff (not ReNs) changed study products weekly and as clinically indicated (e.g. interruption of dressing integrity), with education assistance regarding dressing application provided by the ReN. Product replacements/reinforcements, including tape were recorded. An absorbent dressing, such as sterile gauze, was used alternatively independent from the treatment arm temporarily if the CVAD site was bleeding or oozing [5,32], with such use and its duration, recorded.

All other CVAD management procedures were as per hospital policy including the use of $2 \%$ chlorhexidine gluconate in $70 \%$ alcohol for insertion site decontamination during dressing change, frequency and volume of flushing, negative or neutral displacement mechanical valve needleless connectors, intravenous medication administration and administration set changes [33]. Clinical staff undertook blood and CVAD tip cultures on suspicion of infection, as per standard hospital and pathology protocols $[32,36,37]$.

\section{Statistical Analyses}

Descriptive statistics (counts, percentages) were used to ascertain the primary outcome of feasibility for the 
larger trial. All randomized patients were analyzed on an Intention to Treat (ITT) basis [38]. Comparability of groups at baseline was described across demographic, clinical and device characteristics. Incidence rates (IR) of CVAD device failure and complication (per 1,000 catheter days) were used to summarize the impact of the intervention; with differences evaluated by calculating 95\% confidence intervals.

Kaplan-Meier survival curves (with log rank test) were used to compare CVAD failure, complication, and first dressing duration over time. Standard data cleaning of outlying figures, missing, and implausible data was undertaken prior to analysis. Missing values were not imputed. $\mathrm{P}$ values of $<0.1$ were evaluated as indicating some evidence against a null hypothesis, and values $<0.05$ were considered statistically significant. Stata [39] was used for all analyses.

\section{Ethics}

Ethics approval for the trial was gained from the Children's Health Services Queensland (HREC/13/ $\mathrm{QRCH} / 181$ ) and Griffith University (NRS/10/14/ HREC) Human Research Ethics Committees (HREC). Written informed consent from parents or legal guardians was gained prior to enrolment. Child assent was also gained, where appropriate.

\section{Results}

The study recruited for 21 months, commencing at the Royal Children's Hospital, Brisbane in April 2014 until pausing recruitment in October 2014 due to the merger of two pediatric hospitals, and relocation to a new hospital. Recruitment recommenced at the Lady Cilento Children's Hospital, Brisbane in March 2015, completing recruitment in May 2016 when sample size was achieved. The CONSORT flow chart [40] is displayed in Fig. 2, demonstrating enrolment, allocation, follow-up and analysis of the study participants. Ninety-six patients were screened for recruitment, with 68 eligible, and 48 children participating in the pilot study.

\section{Characteristics}

The majority of participants were undergoing treatment for oncological or hematological conditions $(\mathrm{n}=39$; $81 \%$ ), with a median age of 5.0 years (IQR 1.8, 11.6) (see Table 1). CVADs were most commonly placed in the internal jugular vein $(\mathrm{n}=29 ; 60 \%)$, requiring only a single insertion attempt $(\mathrm{n}=44 ; 94 \%)$. The majority received chemotherapy $(\mathrm{n}=34 ; 71 \%)$, and around half received antibiotics $(n=23 ; 48 \%)$ during the study period. The majority of participant, CVAD insertion and CVAD utility characteristics were evenly distributed across the intervention groups, with imbalance evident in the

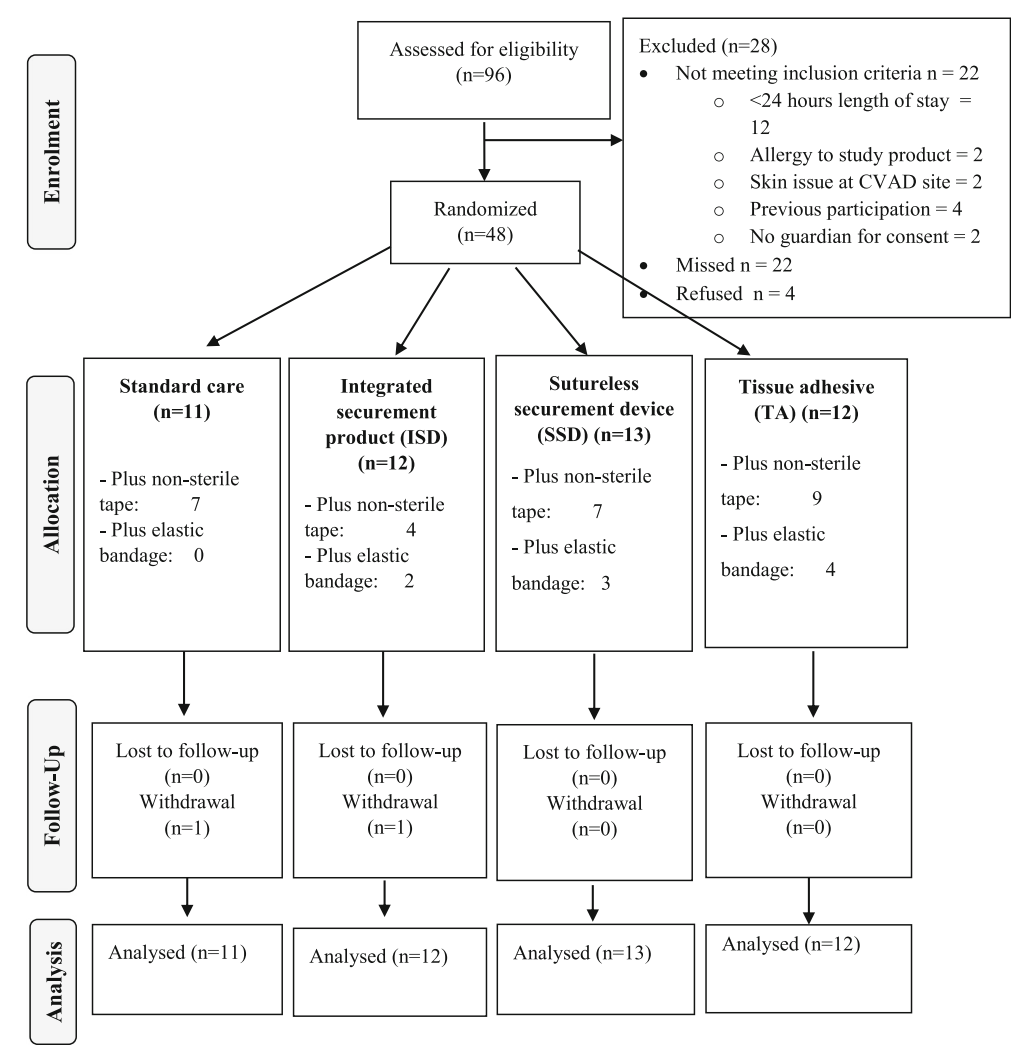

Fig. 2 CONSORT flow chart of study participants 
Table 1 Participant characteristics $(n=48)$

\begin{tabular}{|c|c|c|c|c|c|}
\hline & Standard Care & ISD & TA & SSD & Total \\
\hline Group size & $11(23 \%)$ & $12(25 \%)$ & $12(25 \%)$ & $13(27 \%)$ & 48 (100\%) \\
\hline \multicolumn{6}{|l|}{ Participant characteristics } \\
\hline \multirow[t]{2}{*}{ Age (years) ${ }^{a}$} & 3.1 & 6.2 & 4.9 & 5.0 & 4.8 \\
\hline & $(1.7-14.5)$ & $(1.7-11.7)$ & $(1.0-11.6)$ & $(2.5-8.8)$ & $(1.8-11.6)$ \\
\hline Sex (male) & $7(64 \%)$ & $4(33 \%)$ & $8(67 \%)$ & $10(77 \%)$ & $29(60 \%)$ \\
\hline \multicolumn{6}{|l|}{ Skin integrity: } \\
\hline good & $3(27 \%)$ & $4(33 \%)$ & $7(58 \%)$ & $7(54 \%)$ & $21(44 \%)$ \\
\hline fair & $6(55 \%)$ & $5(42 \%)$ & $4(33 \%)$ & $4(31 \%)$ & 19 (40\%) \\
\hline poor & $2(18 \%)$ & $3(25 \%)$ & $1(8 \%)$ & $2(15 \%)$ & $8(17 \%)$ \\
\hline Skin type (white) & $8(73 \%)$ & $6(50 \%)$ & $10(83 \%)$ & $8(62 \%)$ & $32(67 \%)$ \\
\hline \multicolumn{6}{|l|}{ Comorbidities: } \\
\hline none & $1(9 \%)$ & $0(0 \%)$ & $1(8 \%)$ & $1(8 \%)$ & $3(6 \%)$ \\
\hline one & $7(64 \%)$ & $8(67 \%)$ & $8(67 \%)$ & $9(69 \%)$ & $32(67 \%)$ \\
\hline two or more & $3(27 \%)$ & $4(33 \%)$ & $3(25 \%)$ & $3(23 \%)$ & $13(27 \%)$ \\
\hline \multicolumn{6}{|l|}{ Diagnosis: } \\
\hline oncology / hematology & $8(73 \%)$ & $10(83 \%)$ & $10(83 \%)$ & $11(85 \%)$ & $39(81 \%)$ \\
\hline medical & $2(18 \%)$ & $1(8 \%)$ & $1(8 \%)$ & $1(8 \%)$ & $5(10 \%)$ \\
\hline surgical & $1(9 \%)$ & $1(8 \%)$ & $1(8 \%)$ & $1(8 \%)$ & $4(8 \%)$ \\
\hline Infection on recruitment & $1(9 \%)$ & $2(17 \%)$ & $2(17 \%)$ & $1(8 \%)$ & $6(12 \%)$ \\
\hline Leucocytes $<1,000 / \mu \mathrm{L}$ & $0(0 \%)$ & $3(25 \%)$ & $1(8 \%)$ & $0(0 \%)$ & $4(8 \%)$ \\
\hline \multicolumn{6}{|l|}{ CVAD insertion characteristics } \\
\hline \multicolumn{6}{|l|}{ CVAD placement: } \\
\hline internal jugular & $9(82 \%)$ & $7(58 \%)$ & $7(58 \%)$ & $6(46 \%)$ & $29(60 \%)$ \\
\hline subclavian & $2(18 \%)$ & $4(33 \%)$ & $5(42 \%)$ & $7(54 \%)$ & $18(38 \%)$ \\
\hline femoral & $0(0 \%)$ & $1(8 \%)$ & $0(0 \%)$ & $0(0 \%)$ & $1(2 \%)$ \\
\hline Subsequent insertion & $4(36 \%)$ & $7(58 \%)$ & $5(42 \%)$ & $4(31 \%)$ & $20(42 \%)$ \\
\hline \multicolumn{6}{|l|}{ No. of lumens: } \\
\hline one & $2(18 \%)$ & $0(0 \%)$ & $2(17 \%)$ & $0(0 \%)$ & $4(8 \%)$ \\
\hline two & $8(73 \%)$ & $9(75 \%)$ & $8(67 \%)$ & $11(85 \%)$ & $35(75 \%)$ \\
\hline three & $1(9 \%)$ & $3(25 \%)$ & $2(17 \%)$ & $2(15 \%)$ & $8(17 \%)$ \\
\hline Multiple insertion attempts & $0(0 \%)$ & $0(0 \%)$ & $0(0 \%)$ & $3(23 \%)$ & $3(6 \%)$ \\
\hline Ultrasound use & $4(36 \%)$ & $7(58 \%)$ & $4(33 \%)$ & $4(31 \%)$ & $19(40 \%)$ \\
\hline External length at insertion $(\mathrm{cm})^{b}$ & $12.3(2.5)$ & $15.4(7.5)$ & $13.3(3.9)$ & $13.3(2.0)$ & $13.6(4.5)$ \\
\hline \multicolumn{6}{|l|}{ CVAD utility characteristics } \\
\hline 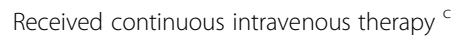 & $4(36 \%)$ & $1(8 \%)$ & $5(42 \%)$ & $6(46 \%)$ & $16(33 \%)$ \\
\hline Received parenteral nutrition and/or lipids ${ }^{c}$ & $4(36 \%)$ & $4(33 \%)$ & $1(8 \%)$ & $2(15 \%)$ & $11(23 \%)$ \\
\hline Received chemotherapy ${ }^{c}$ & $9(82 \%)$ & $8(67 \%)$ & $7(58 \%)$ & $10(77 \%)$ & $34(71 \%)$ \\
\hline Received blood products $^{c}$ & $4(36 \%)$ & $4(33 \%)$ & $2(17 \%)$ & $6(46 \%)$ & $16(33 \%)$ \\
\hline Received antibiotics ${ }^{c}$ & $6(55 \%)$ & $7(58 \%)$ & $1(8 \%)$ & $9(69 \%)$ & $23(48 \%)$ \\
\hline Confused, agitated or drowsy ${ }^{d}$ & $1(9 \%)$ & $3(25 \%)$ & $0(0 \%)$ & $0(0 \%)$ & $4(8 \%)$ \\
\hline \multirow[t]{2}{*}{ CVAD dwell time (days) ${ }^{a, c}$} & 14.1 & 9.0 & 8.6 & 17.1 & 12.4 \\
\hline & $(5.0-27.9)$ & $(4.5-16.5)$ & $(4.1-21.5)$ & $(9.0-27.0)$ & $(5.6-26.0)$ \\
\hline
\end{tabular}

$\mathrm{n}(\%)$ shown unless otherwise noted

CVAD Central venous access device, ISD Integrated securement dressing, SSD Suture-less securement device, TA Tissue adhesive, $\mu L$ microlitre

${ }^{a}$ median (25th and 75 th percentiles); percentages may not add up to $100 \%$ due to rounding; percentages were calculated using the number of non-missing values in the denominator

${ }^{\mathrm{b}}$ mean and standard deviation

c during study period

d at study completion 
frequency of multiple insertion attempts and other characteristics, which is consistent with pilot trial design.

\section{Feasibility of the study process}

As displayed in Fig. 2, the majority of feasibility criteria were met with $71 \%$ of patients screened eligible, with 22 of these patients missed for recruitment due to CVAD insertion scheduling (weekend, out of hours), and 92\% of patients approached for consent agreed to enroll. There were no participants lost to follow up and two patients (4\%) withdrew prematurely from the study. Both of these patients withdrew due to skin irritation (itchiness, redness) associated with study products (standard care; ISD). With parental consent, withdrawn participants were included in the analysis due to their prolonged participation leading to the point of withdrawal. Nine participants (19\%) deviated from the study protocol for their allocated treatment, however for only $4 \%$ of the total studied catheter days (25/647). No primary or secondary outcome data were missed during the study period, and four daily checks were missed out of total $673(0.6 \%)$.

\section{CVAD failure and complications}

Six percent of participants $(n=3)$ experienced CVAD failure prior to completion of treatment (IR 4.46 per 1,000 catheter days; $95 \%$ CI 1.44-13.8), and $6 \%$ of participants $(n=3)$ had a CVAD-associated complication during the study period (IR 4.46 per 1,000 catheter days; 95\% CI 1.44-13.8). As described in Table 2, CVAD failure was highest in the ISD group $(n=2 ; 17 \%)$, and CVAD complications were highest in the SSD group $(n=2 ; 15 \%)$. The TA and standard care groups performed best across both of these criteria with no associated CVAD failures or complications. There were no cases of CVAD-associated thrombosis, complete dislodgement, breakage, local infection, or CVAD-related BSI in any group. CVAD failure results were consistent when failure over time was compared with the Kaplan Meier curve of device failure (Fig. 3).

\section{Staff and parental feedback}

Overall satisfaction was highest in the integrated securement-dressing (mean 8.5/10; SD 1.2). As described in Table 2, mean staff satisfaction with product application (8.8/10; SD 1.6); and removal (8.9; SD 1.6) was highest for standard care; however mean parent satisfaction at study completion was highest for the ISD arm (9.2/10; SD 1.0).

\section{Dressing and securement performance}

Table 3 reports the dressing integrity performance between the study groups. Median time to first dressing change was longest for ISD (95.1 h, IQR 56.1-146), as displayed in the Kaplan Meier curve (Fig. 4), reflecting prolonged time to first dressing change for many participants compared to standard care. Non-routine dressing changes were most common in the standard care arm, with 17 non-routine dressing changes in 162 catheter days (99 per 1000 catheter days). Non-routine dressing changes in the standard care arm were most frequently (59\%) due to lifting of the dressing edges.

\section{Costs}

Each of the experimental arms was more expensive than standard care, when only product purchase costs were considered per application (see Table 3).

\section{Discussion}

This is the world's first randomized controlled trial evaluating the effectiveness of tunneled CVAD dressing and securement in pediatrics. The trial included innovative securement strategies that had never been tested on this device type, or within this population. This pilot trial evaluated the feasibility of a large efficacy trial, using pre-determined feasibility criteria, a registered and published trial protocol, and rigorous methods.

As per our a priori definition of feasibility, a large efficacy trial of tunneled, cuffed CVAD securement and dressing in pediatrics, using these intervention arms, is feasible. Study processes were successful, with targets for eligibility, recruitment, retention, attrition, protocol adherence and missing data achieved. The proportion of failure $(6 \%)$ and complication (6\%) have provided point estimates for future interventional studies; and for a $5 \%$ absolute reduction in CVAD failure or complication is to be reached in future efficacy studies $(p=0.05 ; 80 \%$ power [39]), 211 participants would be needed for each intervention group. The slow recruitment rate in the pilot trial (48 participants over 21 months), means that conducting the efficacy RCT in a single site would be difficult, and a multisite trial, involving other tertiary pediatric hospitals, would be necessary.

However, of the three CVAD failures within the trial, one was not directly associated with the securement device. Within the ISD group, a CVAD spontaneously internally displaced into a non-central position. This suggests the definition of failure would need amending, to exclude this form of failure, in future trials. Overall, it is too soon to assess if one CVAD dressing and securement option is superior. At this early stage of trialing, all products appear generally safe and feasible for future testing. Our feasibility outcomes demonstrated that high-quality research examining CVAD securement in this population is achievable; however the best product for future research is yet to be established.

The most common cause of CVAD-associated complication within the pilot study was partial dislodgement 
Table 2 Study outcomes $(n=48)$

\begin{tabular}{|c|c|c|c|c|c|}
\hline & Standard Care & ISD & TA & SSD & Total \\
\hline Group size & $11(23 \%)$ & $12(25 \%)$ & $12(25 \%)$ & $13(27 \%)$ & $48(100 \%)$ \\
\hline \multicolumn{6}{|l|}{ CVAD failure } \\
\hline Failure & $0(0 \%)$ & $2(17 \%)^{a}$ & $0(0 \%)$ & $1(8 \%)^{b}$ & $3(6 \%)$ \\
\hline Catheter-days & 162 & 139 & 148 & 224 & 673 \\
\hline Incidence Rate & $0.00(\wedge)$ & 14.4 & $0.00(\wedge)$ & 4.46 & 4.46 \\
\hline$(95 \% \mathrm{Cl})$ & & $(3.61-57.7)$ & & $(0.63-31.7)$ & $(1.44-13.8)$ \\
\hline Log-rank test p-values & referent & 0.147 & $\wedge$ & 0.358 & 0.280 \\
\hline \multicolumn{6}{|l|}{ CVAD complications } \\
\hline All-cause complications ${ }^{c}$ & $0(0 \%)$ & $1(8 \%)$ & $0(0 \%)$ & $2(15 \%)$ & $3(6 \%)$ \\
\hline Incidence Rate & $0.00(\wedge)$ & 7.21 & $0.00(\wedge)$ & 8.92 & 4.46 \\
\hline$(95 \% \mathrm{Cl})$ & & $(1.02-51.2)$ & & $(2.23-35.7)$ & $(1.44-13.8)$ \\
\hline Log-rank test p-values & referent & 0.294 & $\wedge$ & 0.358 & 0.600 \\
\hline \multicolumn{6}{|l|}{ Complication $^{c, d}$ : } \\
\hline CABSI & $0(0 \%)$ & $0(0 \%)$ & $0(0 \%)$ & $1(8 \%)$ & $1(2 \%)$ \\
\hline dislodgement (partial) & $0(0 \%)$ & $1(9 \%)$ & $0(0 \%)$ & $1(8 \%)$ & $2(4 \%)$ \\
\hline occlusion (complete) & $0(0 \%)$ & $0(0 \%)$ & $0(0 \%)$ & $1(8 \%)$ & $1(2 \%)$ \\
\hline All-cause skin complication ${ }^{d, e}$ & $2(18 \%)$ & $2(17 \%)$ & $0(0 \%)$ & $1(8 \%)$ & $5(10 \%)$ \\
\hline \multicolumn{6}{|l|}{ Skin complication d, e: } \\
\hline rash & $1(9 \%)$ & $0(0 \%)$ & $0(0 \%)$ & $1(8 \%)$ & $2(4 \%)$ \\
\hline blister & $1(9 \%)$ & $1(8 \%)$ & $0(0 \%)$ & $0(0 \%)$ & $2(4 \%)$ \\
\hline itchiness & $2(18 \%)$ & $1(8 \%)$ & $0(0 \%)$ & $0(0 \%)$ & $3(6 \%)$ \\
\hline \multicolumn{6}{|l|}{ Staff satisfaction ${ }^{\mathrm{f}, \mathrm{g}}$} \\
\hline on application & $8.8(1.6)$ & $7.9(2.0)$ & $7.9(2.1)$ & $7.4(1.7)$ & $8.0(1.9)$ \\
\hline on removal & $8.9(1.6)$ & $8.6(1.6)$ & $5.5(2.8)$ & $8.2(1.6)$ & $7.8(2.4)$ \\
\hline Parent satisfaction on removal $\mathrm{f,g}$ & $5.3(2.3)$ & $9.2(1.0)$ & $8.0(1.7)$ & $8.5(1.9)$ & $7.8(2.3)$ \\
\hline
\end{tabular}

$\mathrm{n}$ (\%) shown unless otherwise noted

CABSI Catheter associated bloodstream infection, CVAD Central venous access device, Cl confidence interval, ISD Integrated securement dressing, TA Tissue adhesive, SSD Suture-less securement device

$\wedge=$ cannot be calculated

a due to spontaneous internal displacement; partial dislodgement

${ }^{\mathrm{b}}$ due to partial dislodgement and occlusion

c at study completion

d Participants could have more than one complication or skin complication

e during the study period

${ }^{f}$ measured on a 0 (minimum) to 10 (maximum) scale

${ }^{g}$ mean and standard deviation

(2/48; 4\%), occurring in two of the intervention arms (ISD, SSD). The rates of complication and failure evident in our study were lower than the recent meta-analysis of observational studies, which found an average CVAD failure proportion of $25 \%$ [6], but still demonstrate the problems associated with CVAD management in pediatrics. Despite higher complications rates, parent satisfaction was highest for the ISD arm, with TA and SSD also performing better than standard care.

Within this study both TA and ISD showed potential to increase the time between CVAD insertion and first dressing change, and their true effectiveness and costeffectiveness should be further investigated. Minimization of dressing changes during the immediate post-insertion period is an important concern for this population. Pediatric patients are frequently non-compliant, and extremely stressed by CVAD dressing changes. Each dressing disruption also potentially places the patient at increased risk for CVAD-associated infection [41] and accidental dislodgement.

Each of the experimental arms was more expensive than standard care, when only basic product purchase costs were considered per application. Additional costs related to the time of skilled clinicians applying and removing the products were not considered, nor the cost of treating complications and failure. These short-term, immediate costs should be weighed against the potential sequelae, if reductions in dressing change frequency, 


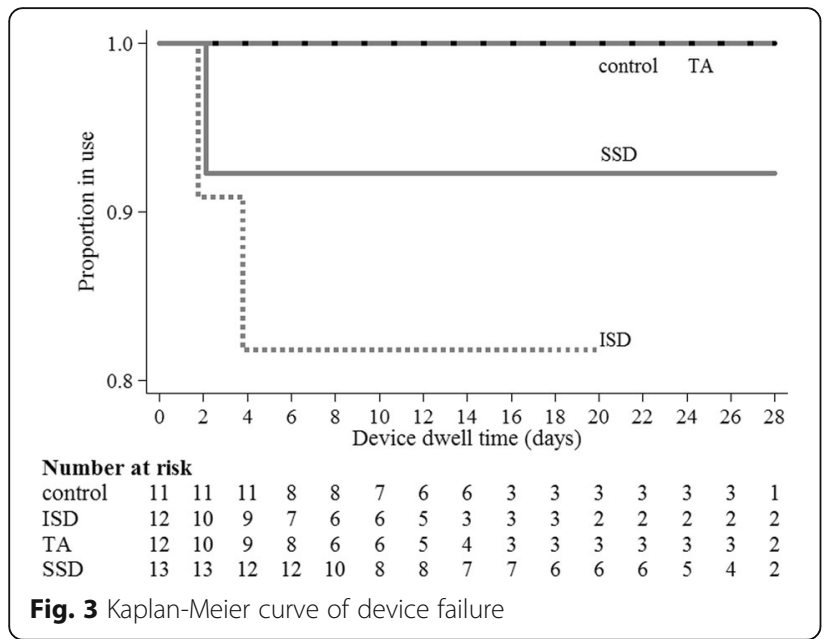

CVAD-associated complications and catheter failure are prevented. Recent case-control studies demonstrated that pediatric CVAD-associated BSIs cost healthcare systems around \$55,646 (2011 USD) and 19 additional days admitted to hospital [42], with even higher costs
(\$69,332; 2011 USD; 21.2 additional days in hospital) for the hematology and oncology pediatric population [43]. Delays to treatment and the insertion of replacement CVADs due to other causes of CVAD failure and complication are also expensive to healthcare systems, and have a significant impact on the morbidity and mortality of pediatric patients. Investment in more expensive CVAD securement devices is likely to be cost-effective if significant improvements in CVAD complication and failure rates can be attained, and future research needs to investigate this.

The recent Cochrane review [9] found moderate quality evidence that chlorhexidine gluconate-impregnated dressing products significantly reduced catheter-related BSI per 1, 000 patient days, and catheter tip colonization, compared with conventional polyurethane dressings. However the majority of these studies were within the adult, critical care population and transferability of these results outside of this population has not been established. Researchers should build on previous work $[12,14]$ to further examine the role of chlorhexidine gluconate-impregnated dressing products in the oncology and pediatric domain.

Table 3 Securement device outcomes $(n=48)$

\begin{tabular}{|c|c|c|c|c|}
\hline & Standard Care & ISD & TA & SSD \\
\hline Group size & $11(23 \%)$ & $12(25 \%)$ & $12(25 \%)$ & $13(27 \%)$ \\
\hline Product purchase costs $^{a}$ & $\$ 5.12-\$ 6.02$ & $\$ 9.27$ & $\$ 17.57-\$ 18.47$ & $\$ 8.60-\$ 12.32$ \\
\hline Patients with dressing changes & $10(91 \%)$ & $8(67 \%)$ & $7(58 \%)$ & $13(100 \%)$ \\
\hline Time to first change (hours) ${ }^{b, c}$ & $\begin{array}{l}79.6 \\
(60.8-104)\end{array}$ & $\begin{array}{l}95.1 \\
(56.1-146)\end{array}$ & $\begin{array}{l}37.0 \\
(11.2-177)\end{array}$ & $\begin{array}{l}61.1 \\
(35.4-111)\end{array}$ \\
\hline \multicolumn{5}{|l|}{ Reasons for first change: ${ }^{d}$} \\
\hline routine & $1(10 \%)$ & $2(25 \%)$ & $3(42 \%)$ & $3(8 \%)$ \\
\hline lifting & $7(70 \%)$ & $2(25 \%)$ & $2(29 \%)$ & $2(15 \%)$ \\
\hline sweating & $1(10 \%)$ & 0 & 0 & 0 \\
\hline leakage & $2(20 \%)$ & 0 & 0 & $1(8 \%)$ \\
\hline skin reaction & $1(10 \%)$ & 0 & 0 & 0 \\
\hline bleeding & $6(60 \%)$ & $4(50 \%)$ & $3(43 \%)$ & $8(61 \%)$ \\
\hline other & $1(10 \%)$ & $2(25 \%)$ & $1(14 \%)$ & 0 \\
\hline Non-routine changes & 17 & 10 & 4 & 25 \\
\hline \multicolumn{5}{|l|}{ Reasons for non-routine changes: ${ }^{d}$} \\
\hline lifting & 10 (59\%) & $2(20 \%)$ & $2(50 \%)$ & $8(32 \%)$ \\
\hline sweating & $1(6 \%)$ & 0 & 0 & 0 \\
\hline leakage & $3(18 \%)$ & 0 & 0 & $1(4 \%)$ \\
\hline skin reaction & $1(6 \%)$ & 0 & 0 & 0 \\
\hline bleeding & $8(47 \%)$ & $6(60 \%)$ & $3(74 \%)$ & 15 (60\%) \\
\hline other & $6(35 \%)$ & $4(40 \%)$ & $1(25 \%)$ & $4(16 \%)$ \\
\hline
\end{tabular}

$\mathrm{n}(\%)$ shown unless otherwise noted

ISD Integrated securement dressing, TA tissue adhesive, SSD Suture-less securement device, $B P U$ bordered polyurethane dressing

${ }^{a}$ in Australian dollars according to local hospital prices 2016

${ }^{b}$ median, 25th and 75th percentiles shown

c excluding when initial securing device did not get replaced

d Participants could have more than one reason 


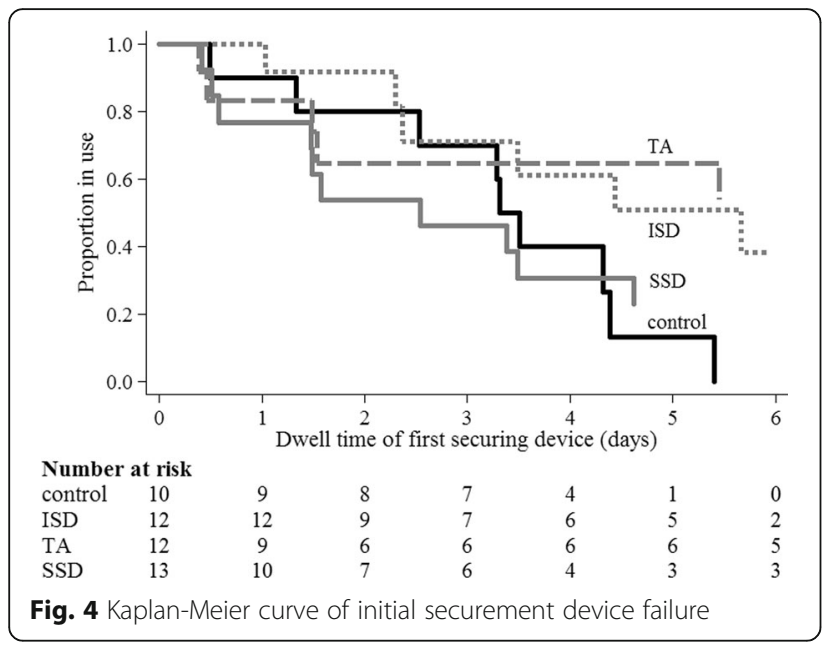

CVAD-associated skin injuries were a substantial issue within our study, with site complications identified in $9 \%$ of participants, with two additional participants $(4 \%)$ withdrawing from the study due to skin irritation. This suggests CVAD-associated skin injuries should be included as a primary, rather than a secondary endpoint in future studies. Pediatric CVAD sites are at risk for significant damage due to the combination of skin impairment related to the patient's age and morbidity, and additional irritation of the CVAD site during maintenance procedures [44]. Local site infection, moisture-related injuries, contact dermatitis and medical adhesive-related injuries (MARSI) related to CVAD sites are frequently reported in the literature [9, 44-48]; however overall prevalence rates have not been published. Identifying patients at risk for skin complication and instituting preventative strategies will further improve clinical outcomes for this vulnerable population.

This pilot study has several limitations. The sample size is small, and the results should not be used to guide clinical practice, however it was never designed to do so. The study was only carried out in two sites in Queensland and its generalizability outside of this population is unknown. Participants, family members and the research staff were not blinded to the intervention allocation, due to the visibility of the securement products. It is highly unlikely that they would intentionally cause CVAD complications because of any preference for any of the treatment arms. The study outcomes of CABSI, catheter-related BSI, and venous thrombosis were assessed by blinded infectious diseases and radiological personnel. The validity and reliability of the study has been ensured through following a prospectively registered study protocol, independent randomization, dedicated ReN, and allocation concealment until study entry.

\section{Conclusions}

High-quality research involving children with tunneled, cuffed CVADs is feasible, and CVAD securement can play an important role in the prevention of CVADassociated complications [9, 49, 50]. Careful consideration should be given by interdisciplinary clinicians when choosing CVAD securement, to ensure it is the most appropriate device for the individual needs of their patient. Further research is necessary to examine the effectiveness of novel securement and dressing products for CVADs in pediatrics.

\section{Abbreviations \\ BPU: Bordered polyurethane; BSI: Bloodstream infection; CABSI: Central venous associated bloodstream infection; Cl: Confidence interval; CONSORT: Consolidated standards of reporting trials; CVAD: Central venous access device; HREC: Human research ethics committee; IQR: Interquartile range; IR: Incidence rate; ISD: Integrated securement device; ITT: Intention to treat; RCT: Randomized controlled trial; SD: Standard deviation; SSD: Suture- less securement device; TA: Tissue adhesive; USD: United States Dollar}

\section{Acknowledgements}

We would like to thank the staff, families and patients of the Royal Children's Hospital, Brisbane and the Lady Cilento Children's Hospital, Brisbane for supporting our research. We would also like to thank the many members of the Alliance for Vascular Access Teaching and Research (AVATAR) group who provided advice in developing this research.

\section{Funding}

This research was funded by the Queensland Health Nursing and Midwifery Early Career Research Fellowship, Centurion Medical Products and Centaur Memorial Fund for Nurses. It has been undertaken as part of Dr Ullman's $\mathrm{PhD}$ program. She has received PhD scholarship funding from the Menzies Health Institute Queensland, NHMRC Centre of Research Excellence in Nursing, the Centaur Postgraduate Scholarship and Centurion Medical Products. The funders have had no role in the study design, collection, analysis and interpretation of the data, writing of the report; and in the decision to submit the paper for publication.

\section{Availability of data and materials}

The dataset supporting these results is not currently available, due to restrictions within the ethical approval process.

\section{Authors' contributions}

All authors have made substantial contributions to the paper and are in agreement with the content. This includes contributions in terms of: 1. the conception and design of the study (AU, TK, CAM, MC, CRM), or acquisition of data (AU, TK, VG), or analysis (AU, GM) or interpretation of data (AU, TK, VG, CAM, MC, CRM), 2. drafting the article or revising critically for important intellectual content (AU, TK, VG, CAM, MC, CRM), and 3. final approval of the version to be submitted (AU, TK, VG, CAM, MC, CRM). All authors read and approved the final manuscript.

\section{Ethics approval and consent to participate}

Ethics approval for the trial was gained from the Children's Health Services Queensland (HREC/13/QRCH/181) and Griffith University (NRS/10/14/HREC) Human Research Ethics Committees (HREC). The trial was also registered with the Australian and New Zealand Clinical Trial Registry (ACTRN12614000280606). Informed consent from parents, legal guardians and children (youth assent, where appropriate) was gained prior to enrolment.

\section{Consent for publication}

Not applicable.

\section{Competing interests}

Griffith University has received research grants to support research projects undertaken by AJU, CRM, and TK from product manufacturers (3M, 
Adhezion, Angiodynamics, Becton Dickinson; BBraun Carefusion; Centurion Medical Products; Entrotech, Teleflex). MC has received an unrestricted educational grant from Baxter.

\section{Publisher's Note}

Springer Nature remains neutral with regard to jurisdictional claims in published maps and institutional affiliations.

\section{Author details \\ 'School of Nursing and Midwifery, Griffith University, Nathan, Queensland, Australia. ${ }^{2}$ Alliance for Vascular Access Teaching and Research Group, Menzies Health Institute Queensland, Nathan, Queensland, Australia. ${ }^{3}$ Children's Health Queensland, Lady Cilento Children's Hospital, South Brisbane, Queensland, Australia. ${ }^{4}$ School of Medicine, University of Queensland, Herston, Queensland, Australia. ${ }^{5}$ School of Medicine, Griffith University, Gold Coast, Queensland, Australia. ${ }^{6}$ Centre for Applied Health Economics, Menzies Health Institute Queensland, Nathan, Queensland, Australia.}

\section{Received: 13 August 2016 Accepted: 24 August 2017}

Published online: 30 August 2017

\section{References}

1. Chopra V, Flanders SA, Saint S, Woller SC, O'Grady NP, Safdar N, Trerotola SO, Saran R, Moureau N, Wiseman S, et al. The Michigan Appropriateness Guide for Intravenous Catheters (MAGIC): Results From a Multispecialty Panel Using the RAND/UCLA Appropriateness Method. Ann Intern Med. 2015;163(6 Suppl):S1-S40.

2. Robinson JL, Casey LM, Huynh HQ, Spady DW. Prospective cohort study of the outcome of and risk factors for intravascular catheter-related bloodstream infections in children with intestinal failure. J Parenter Enteral Nutr. 2013;35(5):625-30.

3. Fratino G, Molinari AC, Parodi S, Longo S, Saracco P, Castagnola E, Haupt R. Central venous catheter-related complications in children with oncological/ hematological diseases: an observational study of 418 devices. Annals of oncology : Official J Eur Soc Med Oncol / ESMO. 2005;16(4):648-54.

4. Barnacle A, Arthurs OJ, Roebuck D, Hiorns MP. Malfunctioning central venous catheters in children: a diagnostic approach. Pediat Radiol. 2008; 38(4):363-78. quiz 486-367

5. O'Grady NP, Alexander M, Burns LA, Dellinger EP, Garland J, O'Heard S, Lipsett PA, Masur H, Mermel LA, Pearson ML, et al. Guidelines for the prevention of intravascular catheter-related infections. Clin Infect Dis. 2011; 52(9):e162-93.

6. Ullman AJ, Marsh N, Mihala G, Cooke M, Rickard C. M.: Complications of central venous access devices: a systematic review. Pediatrics. 2015;136(5): e1331-44.

7. Ullman AJ, Cooke M, Rickard C. Examining the role of securement and dressing products to prevent central venous access device failure: a narrative review. J Assoc Vasc Access. 2015;20(2):99-110.

8. Webster J, Gillies D, O'Riordan E, Sherriff KL, Rickard CM. Gauze and tape and transparent polyurethane dressings for central venous catheters (review). Cochrane Database Syst Rev. 2011;11:1-35.

9. Ullman AJ, Cooke ML, Mitchell M, Lin F, New K, Long DA, Mihala G, Rickard CM. Dressings and securement devices for central venous catheters (CVC). Cochrane Database Syst Rev. 2015;9:Cd010367.

10. Brandt B, DePalma J, Irwin M, Shogan J, Lucke JF. Comparison of central venous catheter dressings in bone marrow transplant recipients. Oncol Nursing Forum. 1996;23(5):829-36.

11. Shivnan JC, McGuire D, Freedman S, Sharkazy E, Bosserman G, Larson E, Grouleff $P$. A comparison of transparent adherant and dry sterile guaze dressings for long-term central catheters in patients undergoing bone marrow transplant. Oncol Nursing Forum. 1991;18(8):1349-56.

12. Chambers ST, Sanders J, Patton WN, Ganly P, Birch M, Crump JA, Spearing RL. Reduction of exit-site infections of tunnelled intravascular catheters among neutropenic patients by sustained-release chlorhexidine dressings: results from a prospective randomized controlled trial. J Hosp Infect. 2005; 61(1):53-61.

13. Olson R, RP HJ, Ryan M, Gilpin J, Falsetti M, Heffner T, Gaudet S. Evaluation of a no-dressing intervention for tunneled central venous catheter exit sites. J Infus Nurs. 2004;27(1):37-44.
14. Ruschulte H, Franke M, Gastmeier P, Zenz S, Mahr K, Bucholz S, Hertenstein $B$, Hecker $\mathrm{H}$, Piepenbrock S. Prevention of central venous catheter related infections with chlorhexidine gluconate impregnated wound dressings: a randomized controlled trial. Ann Hematol. 2009:88:267-72.

15. Harron K, Ramachandra G, Mok Q, Gilbert R. On behalf of the CATCH team: Consistency between guidelines and reported practice for reducing the risk of catheter-related infection in British paediatric intensive care units. Intensive Care Med. 2011;37:1641-7.

16. Yamamoto A, Solomon J, Soulen M, Tang J, Parkinson K, Lin R, Schears G. Sutureless securement device reduces complications of peripherally inserted central venous catheters. J Vascul Int Radiol : JVIR. 2002;13(1):77-81.

17. Wikinson JN, Sheikh N, Jayamaha J. Tissue adhesive as an alternative to sutures for securing central venous catheters. Anaesthesia. 2007;62(9):969-70.

18. Craig P, Dieppe P, Macintyre S, Michie S, Nazareth I, Petticrew M. Developing and evaluating complex interventions: the new Medical Research Council guidance. BMJ. 2008;337:a1655.

19. Ullman AJ, Kleidon T, Gibson V, Long DA, Williams T, McBride CA, Hallahan A, Mihala G, Cooke M, Rickard CM. Central venous Access device SeCurement And Dressing Effectiveness (CASCADE) in paediatrics: protocol for pilot randomised controlled trials. BMJ Open. 2016;6(6)

20. Julious SA. Sample size of 12 per group rule of thumb for a pilot study. Pharmaceutical Stat. 2005;4:287-97.

21. Hertzog M. Considerations in determining sample size for pilot studies. Res Nurs Health. 2008;31:180-91.

22. Lancaster GA, Dodd S, Williamson PR. Design and analysis of pilot studies: recommendations for good practice. J Eval Clin Pract. 2004; 10(2):307-12.

23. Thabane L, Ma J, Chu R, Cheng J, Ismaila A, Rios LP, Robson R, Thabane M, Giangregorio L, Goldsmith CH. A tutorial on pilot studies: the what, why and how. BMC Med Res Meth. 2010;10(1):1.

24. Centers for Disease Control and Prevention. National Healthcare Safety Network Device Associated Module: CLABSI. Atlanta: Edited by Government USOA; 2014. p. 1-9.

25. Fratino G, Avanzini S, Molinari AC, Buffa P, Castagnola E, Haupt R. Incidence of indwelling central venous catheter-related complications using the Sri Paran technique for device fixation in children with cancer. Pediat Surg Int. 2009;25(7):591-4.

26. Broadhurst D, Moureau N, Ullman AJ. Central venous access devices site care practices: an international survey of 34 countries. J Vasc Access. 2015;0(0):0.

27. Cecinati V, Brescia L, Tagliaferri L, Giordano P, Esposito S. Catheter-related infections in pediatric patients with cancer. Eur J Clin Microbiol Infect Dis: Off Pub Eur Soc Clin Microbiol. 2012;31(11):2869-77.

28. Reichman D, Greenberg J. Reducing surgical site infections: a review. Rev Obst Gyn. 2009;2(4):212-21.

29. Advani S, Reich NG, Sengupta A, Gosey L, Milstone AM. Central lineassociated bloodstream infection in hospitalized children with peripherally inserted central venous catheters: extending risk analyses outside the intensive care unit. Clin Infect Dis. 2011;52(9):1108-15.

30. Lorente L, Huidobro MS, Martin MM, Jimenez A, Mora ML. Accidental catheter removal in critically ill patients: a prospective and observational study. Crit Care. 2004;8(4):R229-33.

31. Jumani K, Advani S, Reich NG, Gosey L, Milstone AM. Risk factors for peripherally inserted central venous catheter complications in children. JAMA Pediat. 2013;167(5):429-35.

32. Centre for Healthcare Related Infection Surveillance and Prevention \& Tuberculosis Control: Guideline: Tunnelled Central Venous Catheter. In. Edited by Health Do, version 2 edn. Brisbane: Queensland Government; 2013.

33. Children's Health Services. In: Health Q, editor. Central venous catheters: nursing care and management of tunnelled (cuffed and non-cuffed) CVC in paediatric patients. 1st ed. Brisbane: Queensland Government; 2011.

34. Children's Health Services. CVAD Insertion and Management. Brisbane: Queensland Government; 2014.

35. Frasca D, Dahyot-Fizelier C, Mimoz O. Prevention of central venous catheter-related infection in the intensive care unit. Critical Care. 2010;14(212) doi:10.1186/cc8853.

36. Maki DG, Kluger DM, Crnich CJ. The risk of bloodstream infection in adults with different intravascular devices: a systematic review of 200 published prospective studies. Mayo Clin Proc. 2006:81(9):1159-71.

37. Rickard CM, Marsh NM, Webster J, Gavin NC, McGrail MR, Larsen E, Corley A, Long D, Gowardman JR, Murgo M, et al. Intravascular device administration sets: replacement after standard versus prolonged use in hospitalised 
patients-a study protocol for a randomised controlled trial (The RSVP Trial). BMJ Open. 2015;5(2):e007257.

38. Rothwell PM. Factors that can affect the external validity of randomised controlled trials. PLoS clinical trials. 2006;1(1):e9.

39. StataCorp. Stata Statistical Software: Release 12.1. College Station: StataCorp LP; 2014.

40. Schulz KF, Altman DG, Moher D. CONSORT 2010 statement: updated guidelines for reporting parallel group randomized trials. Ann Intern Med. 2010;152(11):726-32.

41. Timsit JF, Bouadma L, Ruckly S, Schwebel C, Garrouste-Orgeas M, Bronchard R, Calvino-Gunther S, Laupland K, Adrie C, Thuong M, et al. Dressing disruption is a major risk factor for catheter-related infections. Crit Care Med. 2012;40(6):1707-14.

42. Goudie A, Dynan L, Brady PW, Rettiganti M. Attributable cost and length of stay for central line-associated bloodstream infections. Pediatrics. 2014; 133(6):e1525-32.

43. Wilson MZ, Rafferty C, Deeter D, Comito MA, Hollenbeak CS. Attributable costs of central line-associated bloodstream infections in a pediatric hematology/oncology population. Am J Infect Control. 2014;42(11):1157-60.

44. Thayer D. Skin damage associated with intravenous therapy: common problems and strategies for prevention. J Infus Nurs. 2012;35(6):390-401.

45. Wall JB, Divito SJ, Talbot SG. Chlorhexidine gluconate-impregnated centralline dressings and necrosis in complicated skin disorder patients. J Crit Care. 2014;29(6):1130.e1131-4.

46. McNichol L, Lund C, Rosen T, Gray M. Medical adhesives and patient safety: state of the science: consensus statements for the assessment, prevention, and treatment of adhesive-related skin injuries. Orthopedic Nurs. 2013;32(5): 267-81.

47. Kutzscher L. Management of irritant contact dermatitis and peripherally inserted central catheters. Clin J Oncol Nurs. 2012;16(2):E48-55.

48. LeBlanc K, Baranoski S. Skin tears: state of the science: consensus statements for the prevention, prediction, assessment, and treatment of skin tears (c). Advances Skin Wound Care. 2011;24(9 Suppl):2-15.

49. Levy I, Katz J, Solter E, Samra Z, Vidne B, Birk E, Ashkenazi S, Dagan O. Chlorhexidine-impregnated dressing for prevention of colonization of central venous catheters in infants and children: a randomized controlled study. Pediatr Infect Dis J. 2005:4(8):676-9.

50. Hill ML, Baldwin L, Slaughter JC, Walsh WF, Weitkamp JH. A silver-alginatecoated dressing to reduce peripherally inserted central catheter (PICC) infections in NICU patients: a pilot randomized controlled trial. J Perinatol : Off J California Perinatal Assoc. 2010;30(7):469-73.

\section{Submit your next manuscript to BioMed Central and we will help you at every step:}

- We accept pre-submission inquiries

- Our selector tool helps you to find the most relevant journal

- We provide round the clock customer support

- Convenient online submission

- Thorough peer review

- Inclusion in PubMed and all major indexing services

- Maximum visibility for your research

Submit your manuscript at www.biomedcentral.com/submit

) Biomed Central 